\title{
Strong Interaction Between Thiol and Silver
} Modified Silica Nanoparticles in the SERS Sensing of Municipal Solid Waste and Tannery Waste Leachate From Groundwater

\author{
Aarthi A \\ Mother Teresa Women's University \\ Umadevi Mahalingam ( $\nabla$ ums10@yahoo.com ) \\ Mother Teresa Women's University https://orcid.org/0000-0002-2685-187X \\ Parimaladevi $\mathbf{R}$ \\ Mother Teresa Women's University \\ Vasanth G.Sathe \\ UGC - DAE Consortium for Scientific Research
}

\section{Research Article}

Keywords: Thiol and silver modified silica NPs, SERS sensor, Tannery Waste, Municipal Solid Waste, Groundwater

Posted Date: September 30th, 2021

DOI: https://doi.org/10.21203/rs.3.rs-926254/v1

License: (c) (1) This work is licensed under a Creative Commons Attribution 4.0 International License. Read Full License 


\section{Abstract}

A novel surface enhanced Raman spectroscopy (SERS) based polyvinylthiol (PVASH) and silver modified silica nanoparticles (PSA NPS) was prepared to actively concentrate different types of landfill leachate in water. PSA NPs were characterized with UV-visible spectroscopy, XRD, TEM and EDX. SERS sensitivity of the PSA NPs was proved with environmentally ignored leachate from municipal solid waste (MSW) landfill. The result shows that, SERS sensing of PSA NPs were improved with the presence of thiol group. Further, PSA NPs exhibited similar vibrational bands of MSW and tannery waste (TW) landfill leachate in their nearest homeland aquifers. Hence, this study provides a novel SERS sensor of PSA NPs in the tile to detect and analyze the environmentally ignored organic and inorganic compounds.

\section{Introduction}

Landfill waste disposal meant a key management strategy in most developed countries, mainly due to its economical and environmental method to deal with solid waste [1]. Generally, there are three of the most common types of landfills in usage. Household trash and rubbish is sent to a municipal solid waste (MSW) landfill [2]. The hazardous waste collected by the respective category of the hazardous waste landfill must design with proper preventive action. Nowadays, the risk of groundwater pollution is the most considerable environmental concerns of the world [1-5]. The major problem of unlined landfills is the generation of leachate containing many toxic contaminates with unflavored smell has great threat to groundwater and surface water [5]. However, people are all routinely exposed to a broad range of pollutants that are present within the environment. But, nanotechnology has tremendous use in detecting and treating the environmental pollutants due to their enhanced physical and chemical properties of their nanomaterials [6].

Since 1970's, surface enhanced Raman scattering (SERS) is a optical spectroscopic analysis technique with potential for highly sensitive detection of molecules. Also, SERS effect has attracted increasing attention from a broad spectrum of science in the detection of single molecules [7]. SERS is an extremly sensitive technique based mainly on the enormous electromagnetic (EM) enhancement that originated from the excitation of the collective oscillations of free electrons in nanostructured noble metal ( $\mathrm{Au}, \mathrm{Ag}$ and $\mathrm{Cu}$ ) surfaces [8]. To achieve the localized electromagnatic enhancement, three important classification of SERS substrate to be synthesize: Such as, rough sufaces, nanoparticle colloids and periodic nanostructures [9]. But, persistiting organic pollutants (POP's) displays a poor affinity towards the noble metal surface due to their small scattering cross section $[10,11]$. Hence, metal nanoparticles are functionlized by using host molecules or cavitans. The utility of $\mathrm{SiO}_{2}(\mathrm{NMs})$ has received much attention in waste water treatment due to their high surface to volume ratio with its bio compatibility [12, 13]. In addition, thiol(-SH) group has the peculiar attention of coordinating with the metal ions and $\mathrm{S}$ in the thiol group enhance the Raman signal with the yield of Ag NPs. Metal-silica NPs are more stable than pure metal NPs because of their modifying geometries [13]. Naidu et al fabricated the $\mathrm{SiO}_{2} @ A g$ for the detection of dye molecules [14]. Jing et al synthesis thiol modified $\mathrm{Fe}_{3} \mathrm{O}_{4} @ \mathrm{Ag}$ NPs as a SERS substrate for the detection of poly aromatic hydrocarbon (PAH) [15]. Shanthil et al discovered the SERS detection 
performance of $\mathrm{Ag} @ \mathrm{SiO}_{2}$ to be the best depends on their distance between the gaps of the silver NPs [16].

The objective of this work is designed to (i) synthesizing polyvinylthiol (PVASH) and silver modified silica nanoparticles (PSA NPs) as a novel substrate (ii) for the synthesize of PSA NPs, Ag NPs, $\mathrm{SiO}_{2}$ NPs and PVASH were synthesized (iii) as synthezied substrate is actively concentrate the MSW landfill leachate and TW leachate in water. Hence, to the best of our knowledge, this is the first work to synthesize the PSA NPs as a SERS substrate to analyze the MSW and TW landfill leachate in groundwater.

\section{Materials And Methods}

\subsection{Materials}

Silver nitrate, trisodium citrate, were purchased from MERCK. All the chemicals were used as received without further purification. Double distilled water was used throughout the experiment.

\subsection{Synthesis of Ag NPs}

Ag NPs were prepared by chemical reduction method. An aqueous solution of $\mathrm{AgNO}_{3}(1 \mathrm{mM})$ was kept under boiling condition and the reducing agent, aqueous solution of tri sodium citrate ( $2 \mathrm{mM})$ was added drop by drop with vigorous stirring until the colorless precursor aliquot were changed into pale yellow color. The yellow color indicates the synthesize of Ag NPs [17] and was confirmed by optical and morphological studies.

\subsection{Synthesis of silver modified silica NPs}

To obtain silicon functionalized $\mathrm{Ag} \mathrm{NPs}, \mathrm{SiO}_{2}$ was prepareed initially by the mixing of TEOS with aqeous ammonia solution under heating with vigours strring. The precipitate of $\mathrm{SiO}_{2}$ were obtained and dried [16]. Finally, $1 \mathrm{mM}$ of $\mathrm{SiO}_{2}$ was mixed with $1 \mathrm{mM}$ of Ag NPs to obtain silicon functionalized Ag NPs.

\subsection{Synthesis of PSA NPs}

To obtain PSA NPs, PVASH were synthesized as per our previous report [18]. After that, a drop of as synthesized PVASH were added with aliquot of silver modified silica NPs. Hence, the final product PSA NPs were obtained with dark yellow color solution and it was confirmed by optical studies.

\subsection{Sample preparation for SERS analysis}

The SERS sample was prepared by mixing $2.5 \mathrm{ml}$ aliquot of PSA NPs with the aqueous solution of an analyte $(1 \mu \mathrm{l})$. Thus, the prepared SERS samples were exposed to the laser for 5 seconds to collect the SERS signal.

\subsection{Leachate samples}


SERS sensing of PSA NPs were extended to analyse the MSW and TW leachate respectively. Further, for the sensing of percolation of landfill leachate in homeland aquifers, four sampling sites groundwater site1 $(\mathrm{G} 1-(75 \mathrm{~m}))$, groundwater site $2(\mathrm{G} 2-(50 \mathrm{~m}))$, groundwater site $3(\mathrm{G} 3(25 \mathrm{~m}))$ were selected near the TW landfill and MSW landfill at Dindigul district, Tamilnadu, India.

\subsection{Characterization}

UV-visible absorption spectra are measured on a Shimadzu UV-1700 pharma spectrometer. X-ray powder diffraction (XRD) data are recorded on a BRUKER advanced $X$-ray diffractometer using CuKa radiation ( $\lambda$ $=1.5406 \AA$ ) operated at $50 \mathrm{kV}$ and $100 \mathrm{~mA}$. The size and roughness are characterized by transmission electron microscope (TEM, JEM 2100 from JEOL Ltd.) and energy dispersive X-ray analysis is obtained on a transmission electron microscope at an accelerating voltage of $200 \mathrm{kV}$. Raman and SERS spectra are carried out with a micro Raman system Jobin Horiba LABRAM - HR Vis (400 - $1100 \mathrm{~nm})$ at $632.8 \mathrm{~nm}$ excitation wavelength.

\section{Results And Discussion}

\subsection{Optical absorption study}

Figure 1(a) shows the surface plasmon resonance (SPR) band of Ag NPs in the UV-visible energy excitation. Figure 1(b) shows the UV-visible spectrum of silver modified Ag NPs. Figure 1a shows the surface plasmon resonance (SPR) at $413 \mathrm{~nm}$ confirms the presence of pure Ag NPs [17]. The silver modified Ag NPs exhibits absorbance band at $408 \mathrm{~nm}$ shown in Fig. 1b. From Fig. 1c, it was observed that the SPR arises from the PSA NPs at $403 \mathrm{~nm}$ with simultaneous broadened band. The changes in the peak position with simultaneous broadening in the intensity due to the dipole - dipole interaction between PSA NPs. The single and strong SPR band in Fig. 1b confirms the formation of spherically shaped Ag NPs through transverse and longitudinal resonances with the silica particles. In Fig. 1b and 1c, a decreased and broadened intensity of SPR band of Ag NPs is due to the increase in the refractive index of the Ag NPs caused by the presence of $\mathrm{SiO}_{2}, \mathrm{PVASH}$ and Mie plasmon resonance excitation from the $\mathrm{Ag}$ NPs [18]. In addition to that, the changes in the peak position with simultaneous broadening in the intensity were because of reducing interparticle distance between the silica and Ag NPs cores [19]. The reduction in the interparticle distances attributes to the creation of more hotspots leads the substrate acts well in electromagnetic enhancements. Hence, as-synthesized PSA NPs can be proved to be a good SERS analyzer. Further, in Fig. 1, the SPR peak is predominant and single; this confirms that the presence of Ag NPs in the solution is in spherical shape was verified by TEM.

\subsection{Structural studies}

Figure 2 shows the XRD pattern of Ag NPs, silver modified Ag NPs and PSA NPs respectively. Figure 2a shows the diffraction pattern of Ag NPs with its diffraction peak at $38.18^{\circ}(111), 44.38^{\circ}(200), 64.50^{\circ}$ 
(220) and $74.44^{\circ}(311)[17,18]$. In Fig. 2b, addition to the diffraction peaks of Ag NPs with characteristics of the diffraction peak between an angle $15^{\circ}$ to $35^{\circ}$, which belongs to amorphous silica, confirms the presence of silica phase in the as synthesized silver modified Ag NPs [22]. This clearly reflects the strong complex formation between them. Figure $2 \mathrm{c}$ represents the presence of PVASH diffraction peaks addition to the silicon and Ag peaks. The diffracted pattern of thiol was observed at $35^{\circ}(180), 41.7^{\circ}(26-2)$, $46.89^{\circ}(28-2)$ and $48^{\circ}(321)$ confirms the presence of SH group [19]. In addition, an average crystallite size of Ag NPs, silver modified Ag NPs and PSA NPs were estimated using Scherrer formula is approximately $17 \mathrm{~nm}, 12 \mathrm{~nm}$ and $13 \mathrm{~nm}$ respectively.

\subsection{Morphological studies}

The morphology of Ag, silver modified silica NPs and PSA NPs was investigated by TEM and shown in Fig. 3. Figure 3a shows the spherical profile of Ag NPs. In Fig. 3b, aggregated Ag NPs were obtained in the presence of $\mathrm{SiO}_{2}$. From the Fig. 3c, PSA NPs shows the immense aggregation of Ag NPs due to the assistance of SH. Figure 3d shows the energy dispersive X-ray spectroscopy (EDAX) analysis represents the appearance of silica and sulfur element in addition to strong Ag peaks confirms the Ag NPs and sulfur were successfully embedded with the $\mathrm{SiO}_{2}$. This has been demonstrated that the assembled nanostructures possess strong surface plasmon coupling, creating amplified electromagnetic fields at the nanogaps $[19,23]$. Hence, Raman signals of analyte (leachate) molecules can be significantly enhanced by keeping them at the nanogaps of PSA NPs [23]. Since, $\mathrm{SiO}_{2}$ possess negative surface charges, $\mathrm{SH}$ group and Ag NPs were strongly bind to the surface of silica through electrostatic interactions [16]. Therefore, SERS afford the interaction mechanism of PSA NPs and the analyte, as well as the strength of $\mathrm{S}-\mathrm{Ag}-\mathrm{O}-\mathrm{Si}-\mathrm{O}-\mathrm{Ag}-\mathrm{S}$ nanostructure. Also, observed conjoined morphology in the PSA NPs is the source of hotspot makes the substrate to be a good SERS analyzer of the targeted leachate.

\subsection{SERS activity of the substrate using MSW leachate}

The active sensing capability of silver modified silica NPs and PSA NPs were studied by selecting MSW landfill leachate as a target molecule. SERS spectrum of targeted leachate from the Ag modified silica NPs and PSA NPs substrates were demonstrated on the Fig. 4a and 4b. This was clearly shows that more number of highly intensed peaks was exhibited from PSA NPs compared to Ag modified silica NPs. This was clearly confirms the presence of SH group increases the sensitivity of Ag modified silica NPs [24, 25]. Hence, the strength of positively charged $\mathrm{S}-\mathrm{Ag}-\mathrm{O}-\mathrm{Si}-\mathrm{O}-\mathrm{Ag}-\mathrm{S}$ assembled nanostructures has proven to its SERS sensing ability by the adsorption of MSW landfill leachate with high sensitivity. This positively charged compact and conjoined PSA NPs with fine roughness which acts as a nucleation sites to the negatively charged organic pollutants were adsorbed onto surface via electrostatic forces. Hence, observation showed that PSA NPs has used for the further sensing of leachate in the nearest water samples. 


\subsection{SERS sensing of MSW landfill leachate in groundwater}

SERS sensing of MSW landfill leachate in groundwater was recorded and shown in Fig. 5. The SERS spectrum of groundwater samples of the nearest sites to the MSW landfill viz., G1, G2 and G3 were shown in Fig. 5a, Fig. 5b and Fig. 5c) respectively. From the observation, vibrational bands of groundwater samples exhibit similar vibrational bands of MSW leachate the region $1691-1664 \mathrm{~cm}^{-1}$, $1622-1618 \mathrm{~cm}^{-1}, 1593-1568 \mathrm{~cm}^{-1}, 1491-1464 \mathrm{~cm}^{-1}, 1430-1428 \mathrm{~cm}^{-1}, 1372-1313 \mathrm{~cm}^{-1}, 1271-$ $1251 \mathrm{~cm}^{-1}, 1131-1182 \mathrm{~cm}^{-1}, 1093-1003 \mathrm{~cm}^{-1}, 979-916 \mathrm{~cm}^{-1}, 880-806 \mathrm{~cm}^{-1}, 778-712 \mathrm{~cm}^{-1}, 682-$ $622 \mathrm{~cm}^{-1}, 578-527 \mathrm{~cm}^{-1}$. SERS of PSA NPs in water represents vibrational frequencies of aromatic hydrocarbon (AH), phenols, phthalates, pesticide and heavy metal. AH in MSW leachate contains the series of benzene derivatives includes toluene, xylene, ethylbenzen, napthalene etc., [26-29]. The presence of $\mathrm{AH}$ were recognized with the vibrational assignments in the region $979-916 \mathrm{~cm}^{-1}$ shows the ring stretch of aromatic hydrocarbon, $\mathrm{CH}$ bend in the region $1131-1182 \mathrm{~cm}^{-1}$ and the ring deformation in the region $682-622 \mathrm{~cm}^{-1}$ revealed the peak of mono aromatic hydrocarbon [26-29]. Phenols are commonly employed worldwide pollutants, they have involved in the contamination of groundwater as endocrine disruptor. Hence, chlorophenol and cresol were recognized with their vibrational assignment as with the characteristics peaks in the region $1388-1313 \mathrm{~cm}^{-1}$ assigned to $\mathrm{C}-\mathrm{H}$ deformation and $\mathrm{CH}_{3}$ symmetric, $1593-1562 \mathrm{~cm}^{-1}$ assigned to the ring stretching, $682-622 \mathrm{~cm}^{-1}$ assigned to $\mathrm{C}-\mathrm{Cl}$ stretching, $1491-1464 \mathrm{~cm}^{-1}$ assigned to $\mathrm{CH}_{3}$ asymmetric, $1372 \mathrm{~cm}^{-1}, 1044-1003 \mathrm{~cm}^{-1}$ assigned to $\mathrm{CH}_{2}$ scissor vibration and the rocking strong band in the region $778-712 \mathrm{~cm}^{-1}$ revealed the presence of phenols. Zane Arp and co-workers [26, 31] reported, symmetric skeletal stretching mode of the phenyl moiety to produce the Fermi doublet with components near 820 and $842 \mathrm{~cm}^{-1}$ in cresol liquid and strong hydrogenbond acceptance by the phenolic oxygen atom of $\mathrm{p}$-cresol were diagnosed with the canonical maximum value

$I_{2} / I_{1}=2.5[31]$

$I_{2}$ and $I_{1}$ are the peak intensities of the higher and lower wave number of the doublet respectively. From the Fig. 5, SERS spectrum of PSA NPs in water resembles the symmetric stretching mode of phenyl moiety near $820 \mathrm{~cm}^{-1}$ and $840 \mathrm{~cm}^{-1}$ recognized the cresol presence and strong hydrogen bond of phenolic oxygen atom is not involved describe the absence of $p$-cresol. Existence of phthalates in ground water samples was confirmed by the vibrational bands of $\mathrm{NH}$ deformation in the region $1626-1637 \mathrm{~cm}^{-1}$ [32]. The vibrational bands of ring mode with $\mathrm{C}-\mathrm{Cl}$ stretching, ring mode 14 with $\mathrm{CH} 2$ twisting and ring stretching with sharp band in the region $806-880 \mathrm{~cm}^{-1}, 1593-1568 \mathrm{~cm}^{-1}$ and $1271-1251 \mathrm{~cm}^{-1}$ were confirmed the presence of pesticide (simazine) in the ground water [30]. But, the most expected $\mathrm{v}_{\mathrm{s}}(\mathrm{Cr}-\mathrm{O})$ vibrational band of $\mathrm{Cr}(\mathrm{VI})$ was not exhibited in water.

\subsection{SERS analysis of TW landfill leachate}


Based on the high sensitivity of PSA NPs, leachate of TW landfill was analyzed shown in Fig. 6a. A SERS spectrum of TW waste leachate has shown in Fig. $6 b, 6 c$ and $6 d$ shows the SERS of G1, G2 and G3. In Fig. $6 a$, the major bands in SERS spectrum of leachate located at $1680 \mathrm{~cm}^{-1}, 1618 \mathrm{~cm}^{-1}, 1502 \mathrm{~cm}^{-1}$, $1363 \mathrm{~cm}^{-1}, 1328 \mathrm{~cm}^{-1}, 1170 \mathrm{~cm}^{-1}, 992 \mathrm{~cm}^{-1}, 973 \mathrm{~cm}^{-1}, 804 \mathrm{~cm}^{-1}$, in the region $761-695 \mathrm{~cm}^{-1}$. The band in the region 761-695 $\mathrm{cm}^{-1}$ with very strong (vs) vibration is attributing the $\mathrm{CH}$ out-of plane deformation of mono benzenes [26-29]. The band at $738 \mathrm{~cm}^{-1}$ denotes the $-\left(\mathrm{CH}_{2}\right) \mathrm{n}$-of hydrocarbons presence in the leachate [26-29]. The strong (s) band at $761 \mathrm{~cm}^{-1}$ attribute the $\mathrm{NH}_{2}$ wag in amines [2629]. According to the literature by jing et al., the presence of chromium (VI) in the TW leachate has analytically identified with the Raman band at $804 \mathrm{~cm}^{-1}$ [26-29]. Aromatic amines were observed at $1170 \mathrm{~cm}^{-1}$ with "vs" vibration and its $\mathrm{COO}$ group was vibrated in the region $1363-1328 \mathrm{~cm}^{-1}$ (vs) [2629]. Vibrational bands in the region $1585 \mathrm{~cm}^{-1}$ to $1682 \mathrm{~cm}^{-1}$ attributes the $\mathrm{NH}_{2}$ and $\mathrm{NH}_{3}$ deformation of amines [26-29]. Hence, the observed vibrational bands in the Fig. 6 concludes the TW leachate and its active concentration in groundwater. Furthermore, the SERS of water samples from the nearest TW landfill leachate exhibited the similar SERS spectrum of TW leachate which confirms the occurrence of leachate in water sites. The observation shows, among the water samples referred here, water samples (G1, G2 and G3) exhibits the exact similar Raman peaks of TW leachate. In general, SERS of TW leachate takes place by the chemisorptions of organic molecules trapped at the hotspots of dimeric junctions. In particular, major pollutant of TW leachate is $\mathrm{Cr}^{6+}$ has expected to bind with PSA NPs in the way of metalligand interaction shows that it can be an good analyte for the metal detection in the practical way $[6,17$, 18].

\section{Conclusion}

A novel SERS substrate PSA NPs was successfully synthesized with the combination of $\mathrm{SiO}_{2} \mathrm{NPs}, \mathrm{Ag}$ NPs and SH molecules. The unique PSA NPs were synthesized with great aggregation with the source of hotspots. The novel PSA NPs were actively concentrated the MSW and TW landfill leachate and the same in water. Besides the detection of leachate by PSA NPs, the role of Ag NPs in the SERS platform was tested with the presence of $\mathrm{SiO}_{2}$ and $\mathrm{SH}$. Result shows the presence of $\mathrm{SH}$ enhanced the SERS activity of silver modified silica NPs provides the more number of vibrational bands with higher intensity of MSW landfill leachate. Thus, PSA NPs further used to analyze the TW leachate and its percolation in water. SERS detection of MSW and TW leachate exhibits with different vibrational bands according to their innards. The observation shows, MSW and TW landfill leachate exhibits more chemical contaminants than TW landfill leachate. The results conclude that unsecured landfill play a major role of causing the environmental pollution. This study points out a synthesizing of new kind of SERS substrate with qualitative analyzes of common environmental pollution.

\section{Declarations}

\section{Acknowledgement}


The authors are thankful to UGC-DAE-CSR (CSR-IC/CSR-93-2014-2015/599), Indore, India and DST-SERB (EMR/2015/000320), New Delhi, India for financial assistance.

\section{References}

1. F. Wang, D.W. Smith, M.G. El-din, REVIEW / SYNTHÈSE Application of advanced oxidation methods for landfill leachate treatment - A review, 427 (2004) 413-427. doi:10.1139/S03-058

2. S. Shenbagarani, Analysis of Quality near the Solid Waste Dumping Site, IOSR-JESTFT.4 (2013) 0105

3. P. Kjeldsen, M.A. Barlaz, A.P. Rooker, A. Baun, A. Ledin, T.H. Christensen, Present and long-term composition of MSW landfill leachate: A review. Crit Rev Environ Sci Technol.. 32, 297-336 (2002). doi:10.1080/10643380290813462

4. W. Zhang, B. Yue, Q. Wang, Z. Huang, Q. Huang, Z. Zhang, Bacterial community composition and abundance in leachate of semi-aerobic and anaerobic landfills. J Environ Sci 23, 1770-1777 (2011). doi:10.1016/S1001-0742(10)60613-4

5. A.O. Adeolu, O.V. Ada, A.A. Gbenga, O.A. Adebayo, Assessment of groundwater contamination by leachate near a municipal solid waste landfill, Afr. J. Environ. Sci. Technol.. 5, 933-940 (2011). doi:10.5897/AJEST11.272

6. K. Kolomaznik, M. Barinova, H. Vaskova, Chromium VI issue in leather waste - A technology for the processing of used leather goods and potential of raman spectroscopy in chromium traces detection. Math Comput Simul 6, 447-455 (2012)

7. R. Karthikeyan, S. Balaji, P. Sehgal, Industrial applications of keratins-a review. J SCI IND RES. 66, 710-715 (2007). doi:10.1016/j.wasman.2015.05.023

8. SCIENCES WASTE MANAGEMENT IN LEATHER WASTE MANAGEMENT IN LEATHER, (2008) 1-118

9. S. Famielec, K. Wieczorek-ciurowa, WASTE FROM LEATHER INDUSTRY. THREATS TO THE ENVIRONMENT, (n.d.)

10. J. Du, C. Jing, Preparation of Fe304@Ag SERS substrate and its application in environmental Cr(VI) analysis. J. Colloid Interface Sci. 358, 54-61 (2011). doi:10.1016/j.jcis.2011.02.044

11. E.R. Mehmet Kahraman, Mullen, Aysun Korkmaz and Sebastian Wachsmann-Hogiu Fundamentals and applications of SERS-based bioanalytical sensing. Nanophotonics 6(5), 831-838 (2017)

12. B.J. Yun, J.E. Kwon, K. Lee, W.-Gun Koh, Highly sensitive metal-enhanced fluorescence biosensor prepared on electrospun fibers decorated with silica-coated silver nanoparticles, Sensors and amp; Actuators: B. Chemical (2018), 52 https://doi.org/10.1016/j.snb.2018.12.096

13. Balasubramaniam, Saravanakumar, a Yeon-Tae Yu,a Yong-Hoon Cho,b Jin-Kyu Yangc and In-Hwan Lee, Energy coupling processes in InGaN/GaN nanopillar light emitting diodesembedded with $\mathrm{Ag}$ and Ag/SiO2 nanoparticlesJ. Mater. Chem. 22, 21749 (2012)

14. Naidu Dhanpal Jayram, S. Sonia, Palaniswamy Suresh Kumar, L. Marimuthu, Yoshitake Masuda D. Mangalaraj, N. Ponpandian, C. Viswanathan and Seeram Ramakrishna, Highly monodispersed Ag 
embedded $\mathrm{SiO}_{2}$ nanostructured thin film for sensitive SERS substrate: growth, characterization and detection of dye molecules RSC Adv., 2015,5, 46229-46239

15. Jingjing Du, C. Jing, Preparation of Thiol Modified $\mathrm{Fe}_{3} \mathrm{O}_{4} @ A$ Ag Magnetic SERS Probe for PAHs Detection and Identification. J. Phys. Chem. C 115(36), 17829-17835 (2011)

16. M. Shanthil, R. Thomas, R.S. Swathi, and K. George Thomas $\mathrm{Ag} @ S i O_{2}$ Core-Shell Nanostructures: Distance-Dependent Plasmon Coupling and SERS Investigation J. Phys.che.lett, 2012, 3, 1459-1464

17. A.Aarthi, M.Umadevi, R.Parimaladevi, G.V.Sathe, Polyvinyl thiol assisted Ag NPs as an efficient SERS analyzer and visible light photocatalyst for tannery waste landfill leachate, j.vacuum 161, 2019, 125129 https://doi.org/10.1016/j.vacuum.2018.12.022

18. A. Aarthi, M. Umadevi, R. Parimaladevi, G.V. Sathe, Detection and degradation of leachate in groundwater using ag modi fi ed Fe 304 nanoparticle as sensor. J. Mol. Liq. 252, 97-102 (2018). doi:10.1016/j.molliq.2017.12.103

19. Y. Gang Chen, M. Wang, J. Yang, S.J. Xu, M. Goh, Pan, H. Chen, Measuring Ensemble-Averaged Surface-Enhanced Raman Scattering in the Hotspots of Colloidal Nanoparticle Dimers and Trimers. J. AM. CHEM. SOC 132, 3644-3645 (2010)

20. P. Fageria, S. Gangopadhyay, S. Pande, Synthesis of $\mathrm{ZnO} / \mathrm{Au}$ and $\mathrm{ZnO} / \mathrm{Ag}$ nanoparticles and their photocatalytic application using UV and visible light. RSC Adv. 4, 24962-24972 (2014). doi:10.1039/C4RA03158J

21. Zamiri R, Azmi BZ, Sadrolhosseini AR, Ahangar HA, Zaidan AW, Mahdi MA, Preparation of silver nanoparticles in virgin coconut oil using laser ablation Int. J Nanomedicine. 6 (2011) 71 - 5. doi: 10.2147/IJN.S14005

22. G.V.MamontovV.V. DutovV. I. Sobolev, O.V. Vodyankina, Effect of transition metal oxide additives on the activity of an $\mathrm{Ag} / \mathrm{SiO}_{2}$ catalyst in carbon monoxide oxidation. Kinet. Catal. 54, 487-491 (2013)

23. J.-M. Nam, J.-W. Oh, Haemi Lee, and Yung Doug Suh, Plasmonic Nanogap-Enhanced Raman Scattering with Nanoparticles, Acc. Chem. Res, DOI: 10.1021/acs.accounts.6b00409

24. J. Bao, Y. Fu, Z. Bao, Thiol-functionalized magnetite / graphene oxide hybrid as a reusable adsorbent for Hg 2 + removal. Nanoscale Res Lett 3, 4-9 (2013)

25. Y. Chen, L. Wu, Y. Chen, N. Bi, X. Zheng, H. Qi, M. Qin, X. Liao, H. Zhang, Y. Tian, Determination of mercury(II) by surface-enhanced Raman scattering spectroscopy based on thiol-functionalized silver nanoparticles. Microchim. Acta 177, 341-348 (2012). doi:10.1007/s00604-012-0777-6

26. F. Wikipedia, Infrared spectroscopy correlation table, J APPL SPECTROSC. (2008) 1-3. doi:10.1063/1.1497629

27. RAMAN Band Correlation Table, University of Toronto Scarborough. (2010) 1-2

28. F. Identifying Groups with Raman Spectroscopy, (1991)

29. Infrared spectroscopy correlation table - Wikipedia, the free encyclopedia, (n.d.)

30. J.C.S. Costa, R.A. Andro, P.H.C. Camargo, P. Corio, Understanding the effect of Adsorption Geometry over Substrate Selectivity in the Surface-Enhanced Raman Scattering Spectra of Simazine and 
Atrazine,J.Phys.Chem.115 (2011) 4184-4190

31. Z. Arp, D. Autrey, J. Laane, S.A. Overerman, G.J. Thomas, Tyrosine Raman Signatures of the Filamentous Virus Ff are Diagnostic of Non- Hydrogen-Bonded Phenoxyl: Spectroscopy of $p$-Creol Vapor. J. Biochem. 40, 2522-2529 (2001)

32. Q. An, P. Zhang, J.M. Li, W.F. Ma, J. Go, J. Hu, C.C. Wang, Silver-coated Magnetite-carbon core-shell microspheres as substrate-enhanced SERS probes for detection of trace persistent organic pollutants,Nanoscale 4 (2012) 5210-5216

Figures



Figure 1

UV-visible spectra of (a) Ag NPs and (b) silver modified silica NPs (c) (PSA-NPs) 


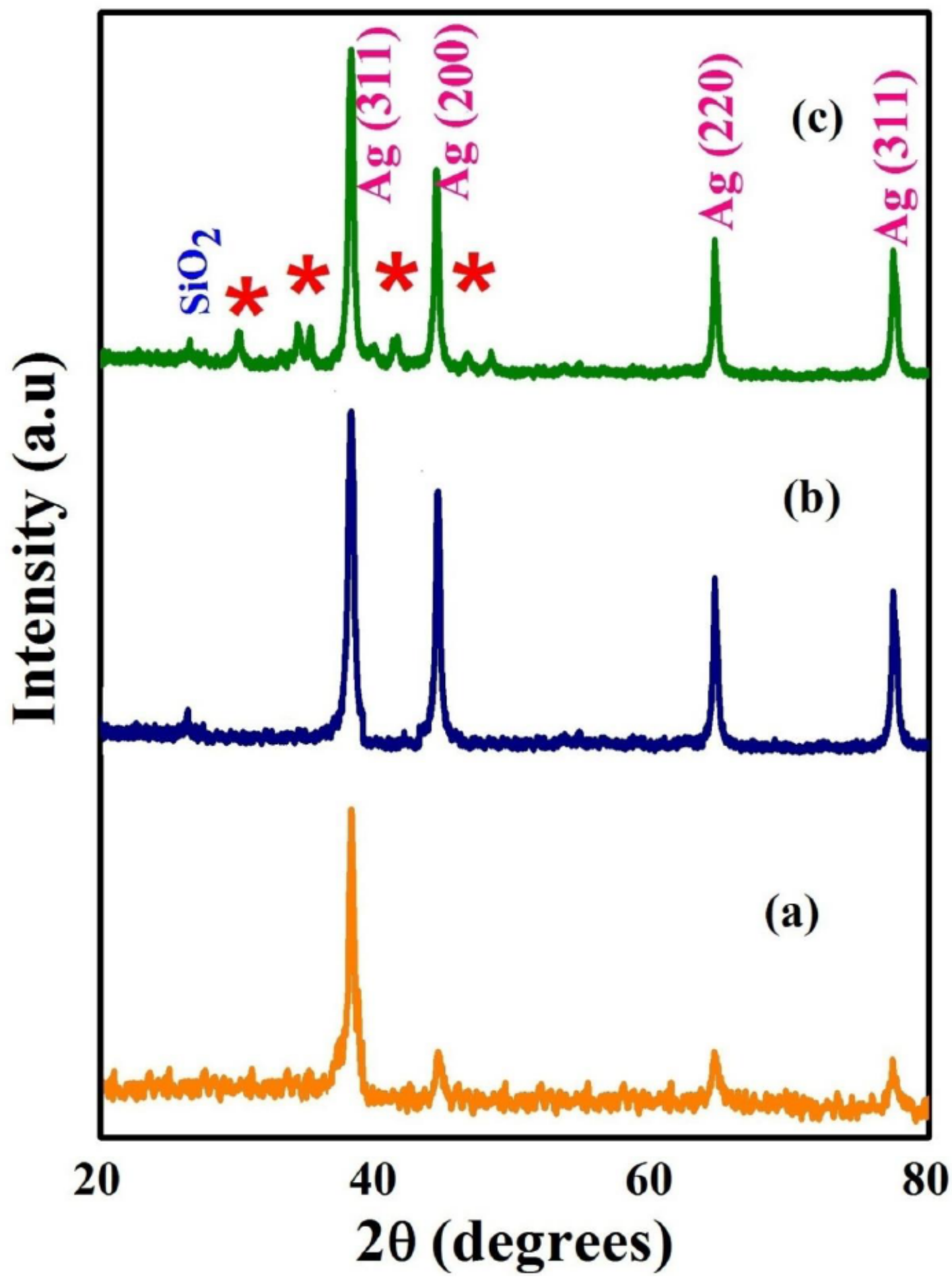

Figure 2

XRD pattern of (a) Ag NPs (b) silver modified silica NPs (c) PSA NPs 


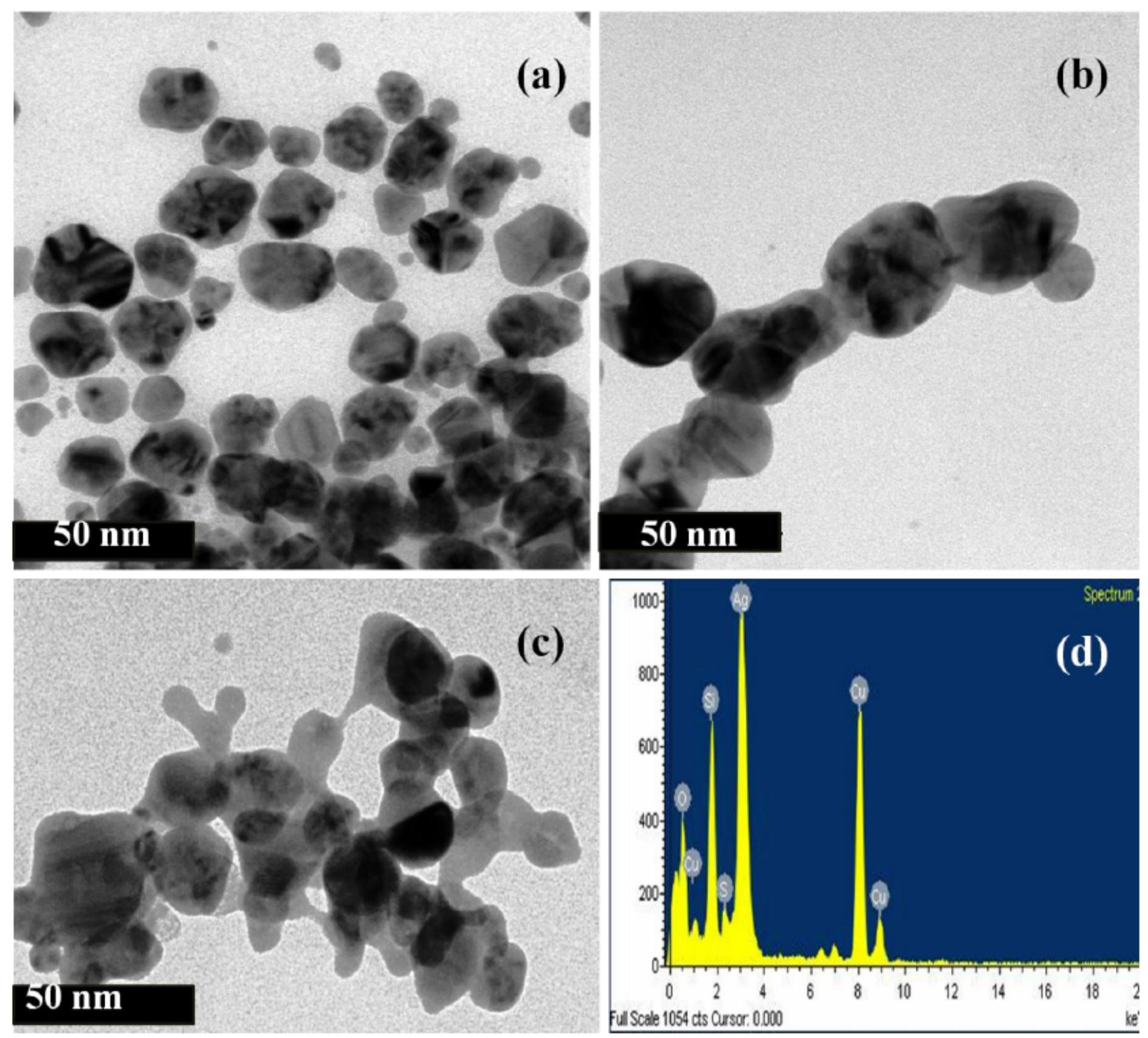

Figure 3

TEM picture of (a) Ag NPs (b) silver modified silica NPs (c) PSA NPs (d) EDX of PSA NPs 

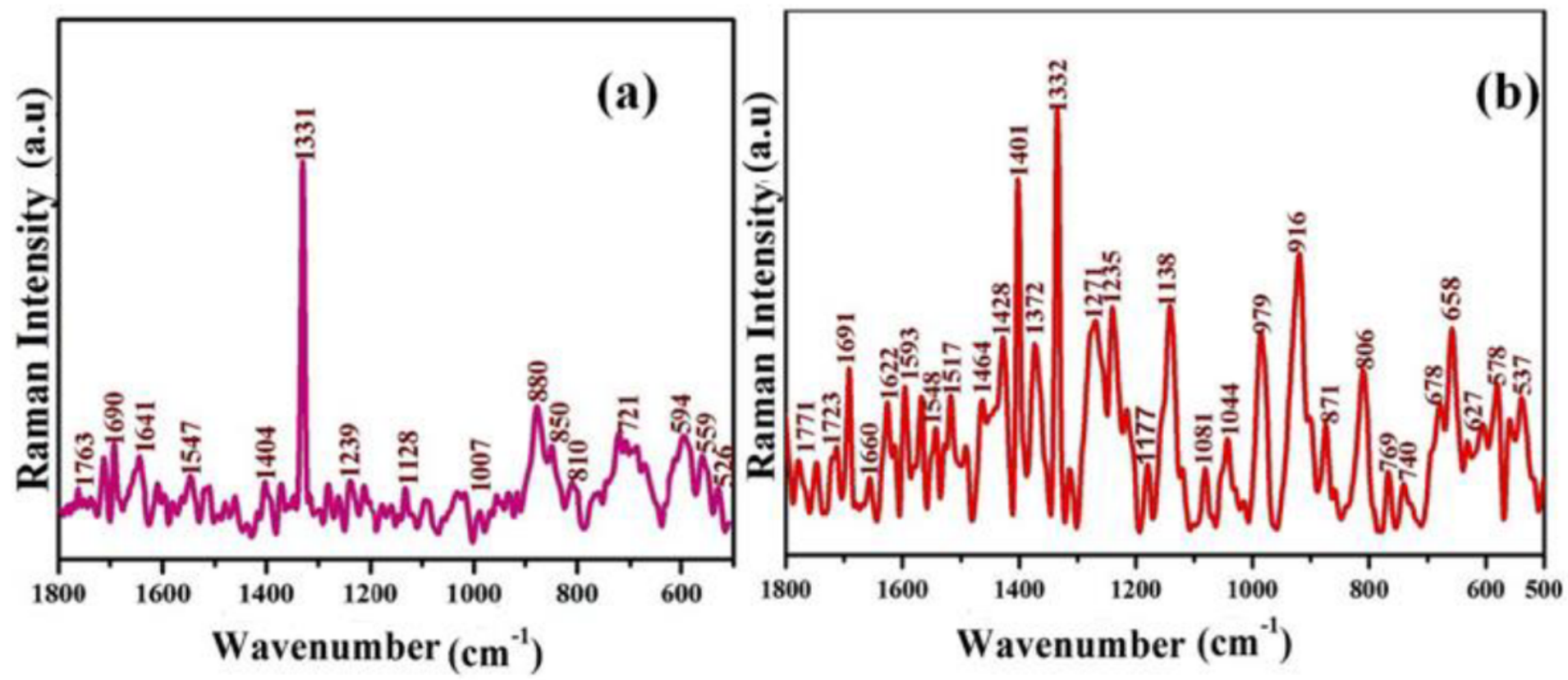

Figure 4

SERS spectrum of MSW leachate by using (a) Silver modified silica NPs (b) PSA NPs 

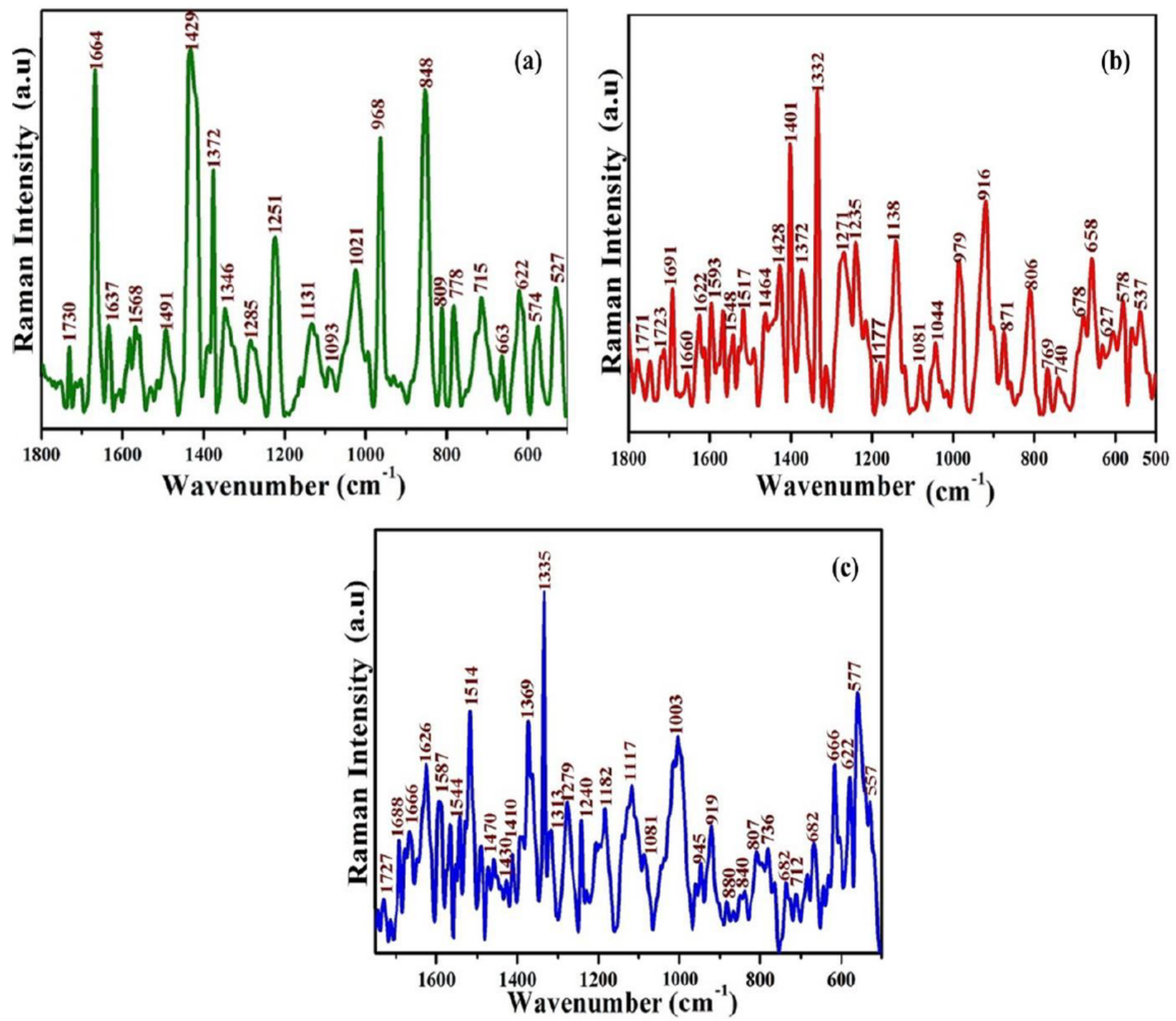

Figure 5

SERS spectrum of leachate in water (a) G1 (b) G2 and (c) (G3) using PSA NPs 


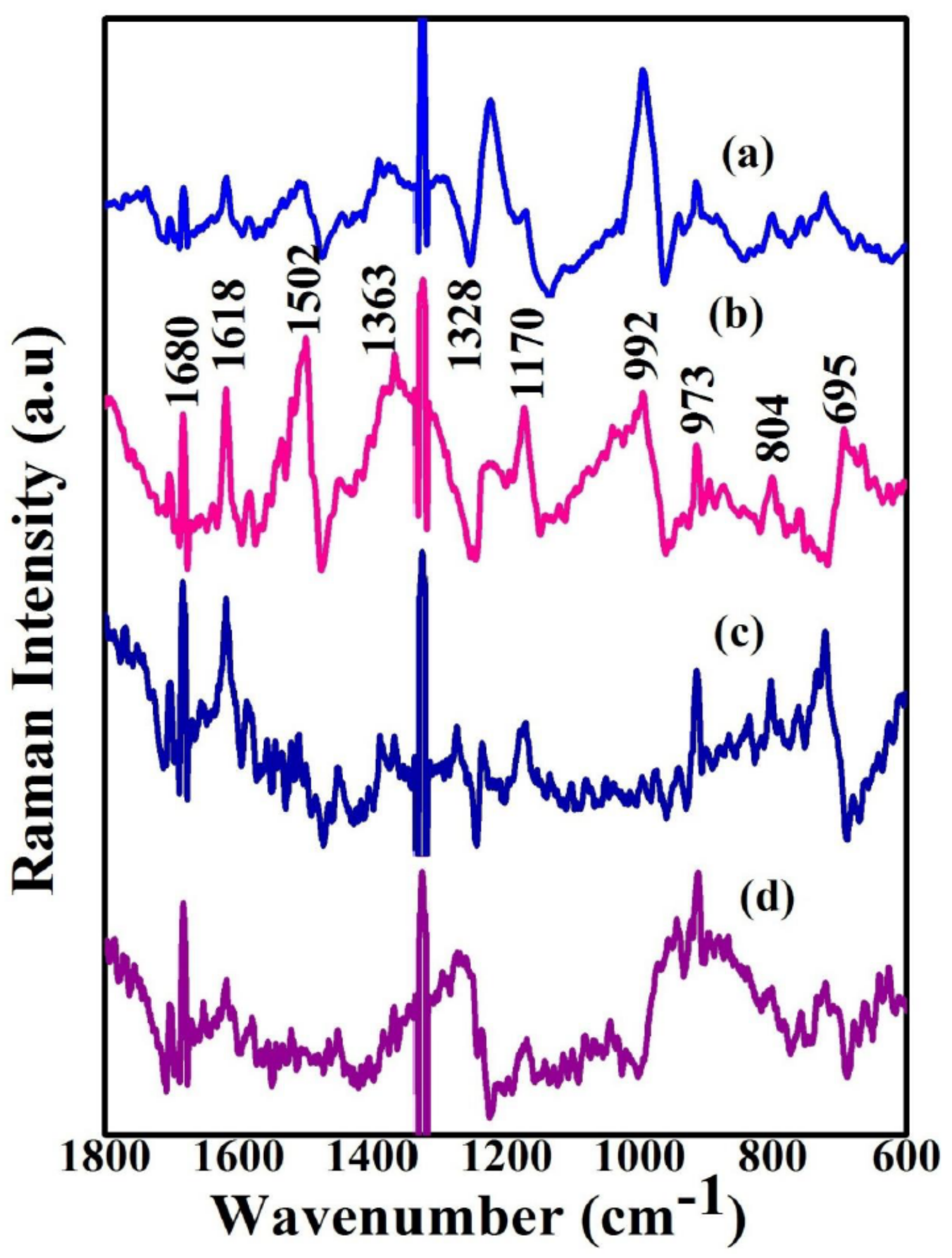

Figure 6

SERS Spectrum of (a) TW landfill leachate (b) G1 (c) G2 (d) G3

\section{Supplementary Files}

This is a list of supplementary files associated with this preprint. Click to download. 
- GraphicalAbstract.png 